Sensors 2003, 3, 69-82

\title{
sensors
}

ISSN 1424-8220

(C) 2003 by MDPI

http://www.mdpi.net/sensors

\section{A Sentinel Sensor Network for Hydrogen Sensing}

Craig A. Grimes ${ }^{1, *}$, Keat G. Ong ${ }^{2}$, Oomman K. Varghese ${ }^{1}$, Xiping Yang ${ }^{1}$, G. Mor ${ }^{1}$, Maggie Paulose $^{2}$, Elizabeth C. Dickey ${ }^{3}$, Chuanmin Ruan ${ }^{1}$, Michael V. Pishko ${ }^{4}$, James W. Kendig ${ }^{5}$ and Andrew J. Mason ${ }^{6}$

${ }^{1}$ Dept. of Electrical Engineering and Materials Research Institute, The Pennsylvania State University, 217 Materials Research Laboratory, University Park, PA 16802.

${ }^{2}$ SenTech Corporation, 200 Innovation Boulevard Suite 236, State College, PA 16803.

${ }^{3}$ Dept. of Materials Science and Engineering, The Pennsylvania State University, University Park, PA 16802.

${ }^{4}$ Dept. of Chemical Engineering, The Pennsylvania State University, University Park, PA 16802.

${ }^{5}$ Division of Newborn Medicine, Penn State Children's Hospital, The Milton S. Hershey Medical Center, 500 University Drive, Hershey, PA 17033

${ }^{6}$ Dept. of Electrical and Computer Engineering, Michigan State University, Lansing, MI

* Author to whom correspondence should be addressed. E-mail: cgrimes@engr.psu.edu

Received: 14 February 2003 / Accepted: 20 February 2003 / Published: 21 February 2003

\begin{abstract}
A wireless sensor network is presented for in-situ monitoring of atmospheric hydrogen concentration. The hydrogen sensor network consists of multiple sensor nodes, equipped with titania nanotube hydrogen sensors, distributed throughout the area of interest; each node is both sensor, and data-relay station that enables extended wide area monitoring without a consequent increase of node power and thus node size. The hydrogen sensor is fabricated from a sheet of highly ordered titania nanotubes, made by anodization of a titanium thick film, to which platinum electrodes are connected. The electrical resistance of the hydrogen sensor varies from $245 \Omega$ at $500 \mathrm{ppm}$ hydrogen, to $10.23 \mathrm{k} \Omega$ at $0 \mathrm{ppm}$ hydrogen (pure nitrogen environment). The measured resistance is converted to voltage, $0.049 \mathrm{~V}$ at $500 \mathrm{ppm}$ to $2.046 \mathrm{~V}$ at $0 \mathrm{ppm}$, by interface circuitry. The microcontroller of the sensor node digitizes the voltage and transmits the digital information, using intermediate nodes as relays, to a host node that downloads measurement data to a computer for display.
\end{abstract}


This paper describes the design and operation of the sensor network, the titania nanotube hydrogen sensors with an apparent low level resolution of approximately $0.05 \mathrm{ppm}$, and their integration in one widely useful device.

Keywords: Sensor network, Sensor node, Sensor array, Hydrogen, Sensor, Nanotube

\section{Introduction}

There is an ongoing need to accurately detect molecular hydrogen $\left(\mathrm{H}_{2}\right)$ for a variety of applications in industrial process control. Such applications include chemical and petroleum refining, rocket fuels, and electric power production via low-emission fuel cells to cite but a few examples. In the production of high-purity gases such as silane and nitrogen for the semiconductor manufacturing industry, or for production of kypton, xenon and neon for the lighting industry, hydrogen is often a contaminant that must be quantified to levels ranging from $0.1 \mathrm{ppm}$ to $100 \mathrm{ppm}$. In the space industry, where hydrogen is used as a rocket fuel, $\mathrm{H}_{2}$ leak detection is a critical safety concern.

Breath hydrogen is an important, clinically relevant parameter used as an indicator of lactose intolerance [1-3], fructose malabsorption [4-8], microbial activity [9], bacterial growth [10-12], fibromyalgia [13], diabetic gastroparesis [14-16], and neonatal necrotizing enterocolitis [17-21]. The pathogenesis of neonatal necrotizing enterocolitis (NEC), a devastating disease due to bacterial fermentation which causes necrosis of the intestinal walls of preterm infants, results in the production of hydrogen gas which accumulates as bubbles in the sub-mucosal area of the bowel wall as shown in Figure 1. Engel et al [18] used a micro-syringe to collect a gas sample from one of these bubbles and, on analysis, found that the gas in the bubble was $30 \%$ hydrogen.

a)

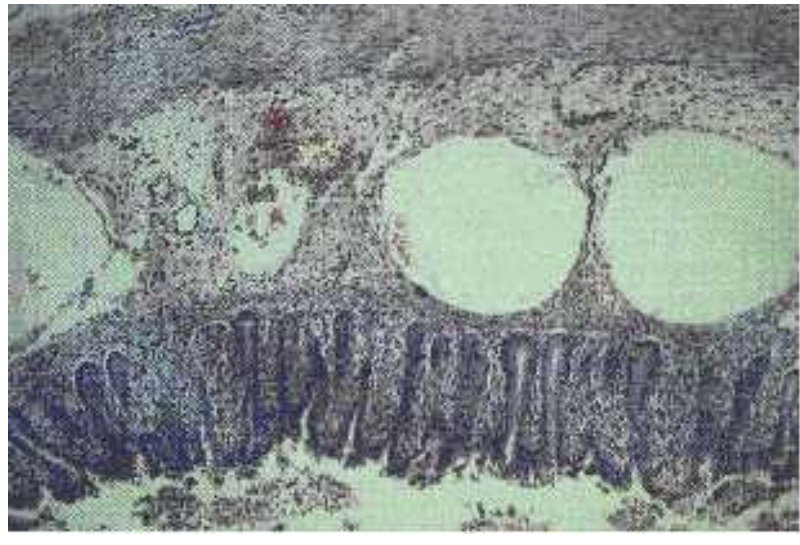

b)

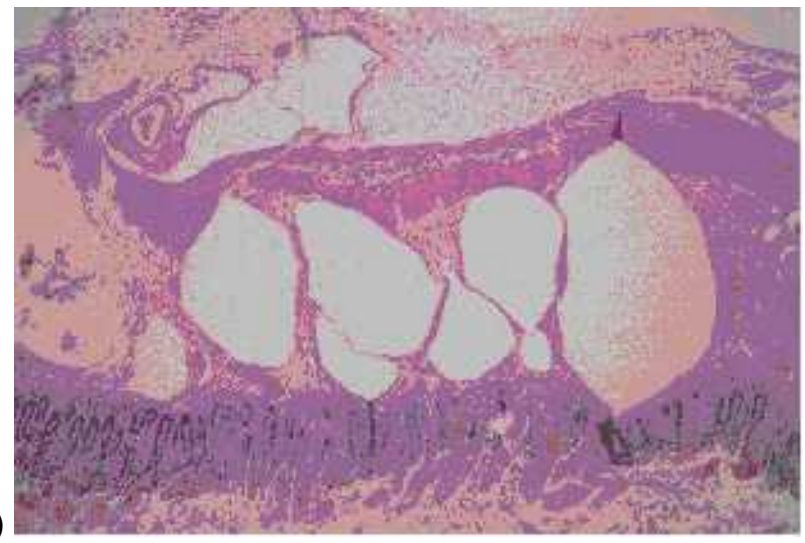

Figure 1. a) Histopath slide of NEC with intact mucosa and large bubbles of hydrogen and nitrogen gas in the submucosal area. b) Histopath slide of advanced NEC with mucosal breakdown and hydrogen and nitrogen gas bubbles in the submucosal area.

Hydrogen is also absorbed into the blood stream and excreted via the lungs into the exhaled breath. Therefore an effective way to detect NEC is by continuously monitoring hydrogen concentrations in 
the exhaled gas from the infant [19-21]. For example Godoy et al [19] measured exhaled breath hydrogen levels in 25 preterm infants with documented NEC (presence of clinical symptoms and pneumatosis on abdominal radiographs) and compared them with levels drawn from similar infants with feeding intolerance, but no NEC. They found that 19 of the 25 infants with NEC had exhaled breath hydrogen levels equal to or greater than $20 \mathrm{ppm}$, while only 2 out of 16 infants with feeding intolerance had levels equal to or greater than $20 \mathrm{ppm}$. While breath concentration in exhaled breath could be a parameter of considerable clinical utility with current methodology it is difficult, time consuming and expensive to collect exhaled gas samples from preterm infants for hydrogen analysis, which requires collected breath samples to be taken to a laboratory for hydrogen analysis using typically a gas chromatograph. For monitoring of NEC in pre-term infants it appears a clinically useful hydrogen sensor must be capable of detecting hydrogen at levels of approximately $25 \mathrm{ppm}$ to $1 \mathrm{ppm}$. Furthermore the packaged hydrogen sensor must be non-invasive since physically disturbing the preterm infants significantly hampers their physical and mental development processes.

In many instances practical application of hydrogen sensors would be significantly enhanced by development of an inexpensive, wide-area sensor network technology incorporating hydrogen sensors capable of real-time in-situ detection of $0.1 \mathrm{ppm}$ to $1000 \mathrm{ppm}_{2}$ detection. As a step towards that ultimate goal, in this work titania-nanotube hydrogen sensors [22,23] are incorporated into a wireless sensor network [24-26] to measure hydrogen concentrations from multiple sources, in real time. The sensor network consists of an array of nodes distributed throughout the area of interest, with a 'host' node connected directly to a computer that graphically presents the collected data. The main component of a sensor node is a microcontroller that oversees node operation, including transmission of node sensor information as well as relaying of information from distant nodes towards nodes closer to base. In this work each node has a RF transceiver integrated with the microcontroller for wireless communication between nodes; in earlier work we described acoustic-wave communication between nodes of an aqueous sensor network [27]. Interface circuitry is used to convert the response of the hydrogen sensor, a change in electrical resistance, to a voltage variation so it can be digitized and processed by the microcontroller. The host node also contains a serial interface to communicate with a computer via RS232 protocol.

The hydrogen sensor is comprised of a highly ordered thin film of titania nanotubes, made using an anodization technique [28,29], to which platinum electrodes are connected. Due to chemisorption of molecular hydrogen the electrical resistance of the titanium dioxide nanotubes is highly sensitive to hydrogen; for example, for $76 \mathrm{~nm}$ diameter nanotubes there is a variation in measured resistance of $10^{3}$ when cycling between nitrogen atmosphere and $500 \mathrm{ppm}$ hydrogen at $290^{\circ} \mathrm{C}$. The hydrogen sensor is also completely reversible, and has a response time of approximately 5 minutes and a recovery time of about 30 minutes. The sensitivity of the nanotubes to hydrogen is highly dependent upon nanotube diameter, with $22 \mathrm{~nm}$ diameter nanotubes (not considered here) being approximately 200 times more sensitive than $76 \mathrm{~nm}$ diameter nanotubes, although the smaller diameter nanotubes have a larger surface area by only $30 \%$. The titania nanotube hydrogen sensors exhibit high selectivity towards hydrogen compared to reducing gases such as carbon monoxide, ammonia and carbon dioxide [30]. In oxygen the electrical resistance of the titania nanotube sensor is reduced by an approximate factor of two, from which it does not recover. 


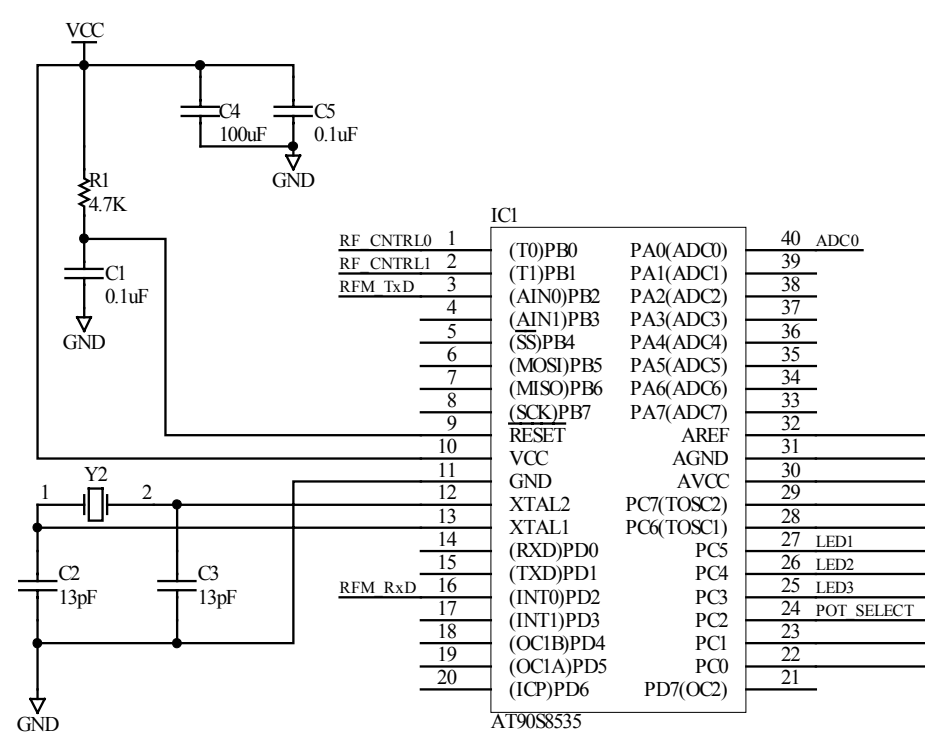

To rest of circuit

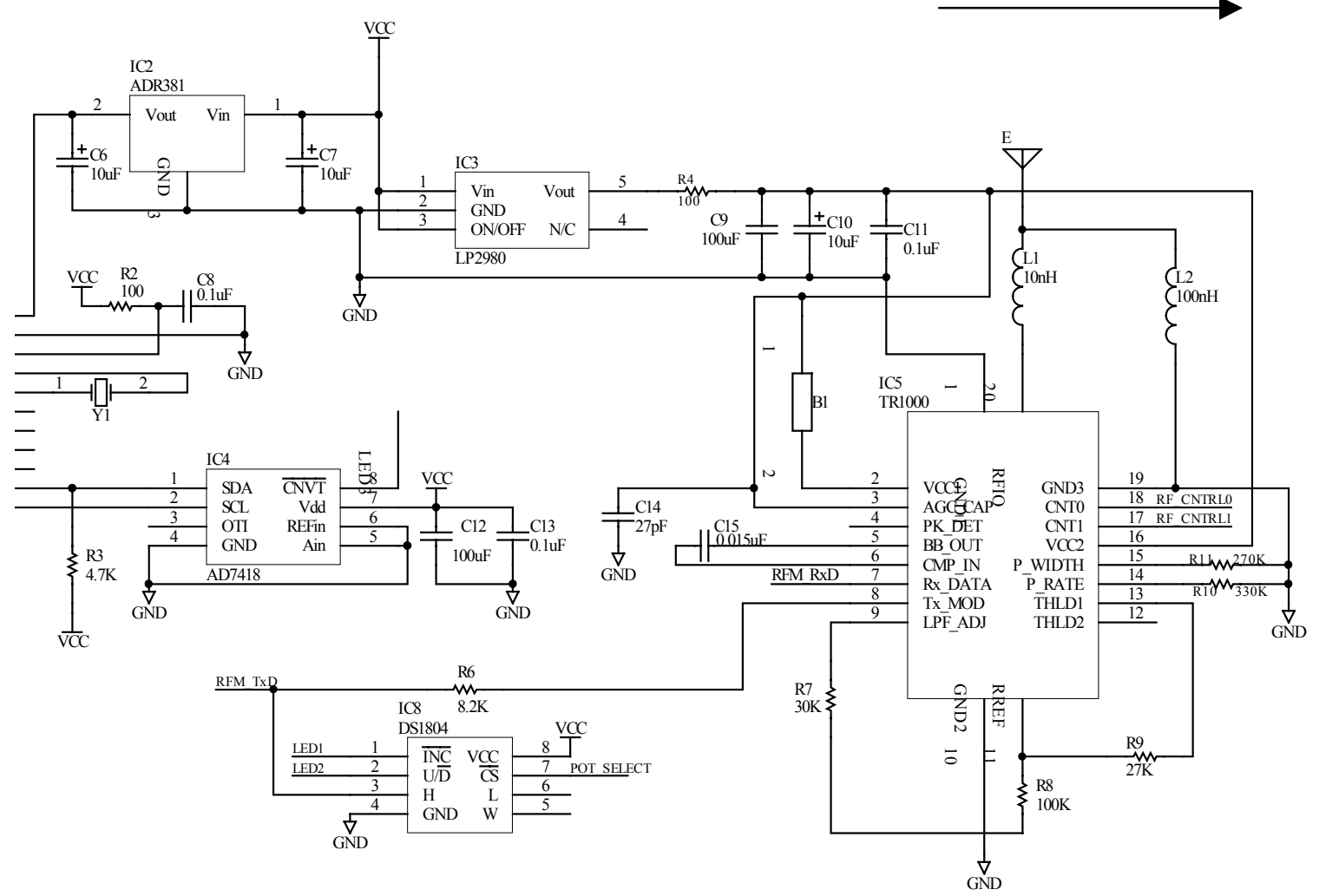

Figure 2. Circuit schematic of the sensor node. The ADC0 port (pin 40) of the microcontroller AT90S8535 is connected to the output of the sensor interface circuit.

\section{Design and Fabrication of Hydrogen Sensor Network}

\section{Wireless Sensor Network Design}

Before a sensor node can transmit data it needs to know the identity of its 'parent,' that is the node it should relay its data to. To achieve this, the computer first instructs the host node to send a broadcast signal that contains its identity. When a nearby node receives the signal, it remembers the host node as 
its parent node, and in turn sends its own broadcast signal to other nodes. This process is repeated until all nodes in the network know their parent node identity. The broadcasting operation is important for the nodes in the network to initialize communication links, or re-establish communications after some nodes are damaged or removed. When relaying sensor information, all nodes transmit data to their parent nodes. This will ensure the data of all nodes will be eventually sent to the host node and downloaded to the host computer. Further details on communication protocols of the sensor network are detailed in an earlier work [27].

The circuit schematic of the sensor node is shown in Fig. 2. The microcontroller (AT90S8535) is connected to a RF transceiver chip (TR1000) via its serial bus. The microcontroller oversees all operations of the node, including acquiring data from the sensor, transmitting sensor data, and relaying the data of other nodes [27]. A temperature sensor is included in each sensor node (Analog Devices AD7418). The resistance of the hydrogen sensor is converted to a voltage by a sensor interface circuit (see Fig. 3). The converted voltage is sent to the analog-to-digital converter port (ADC0, pin 40) of the microcontroller to be digitized.

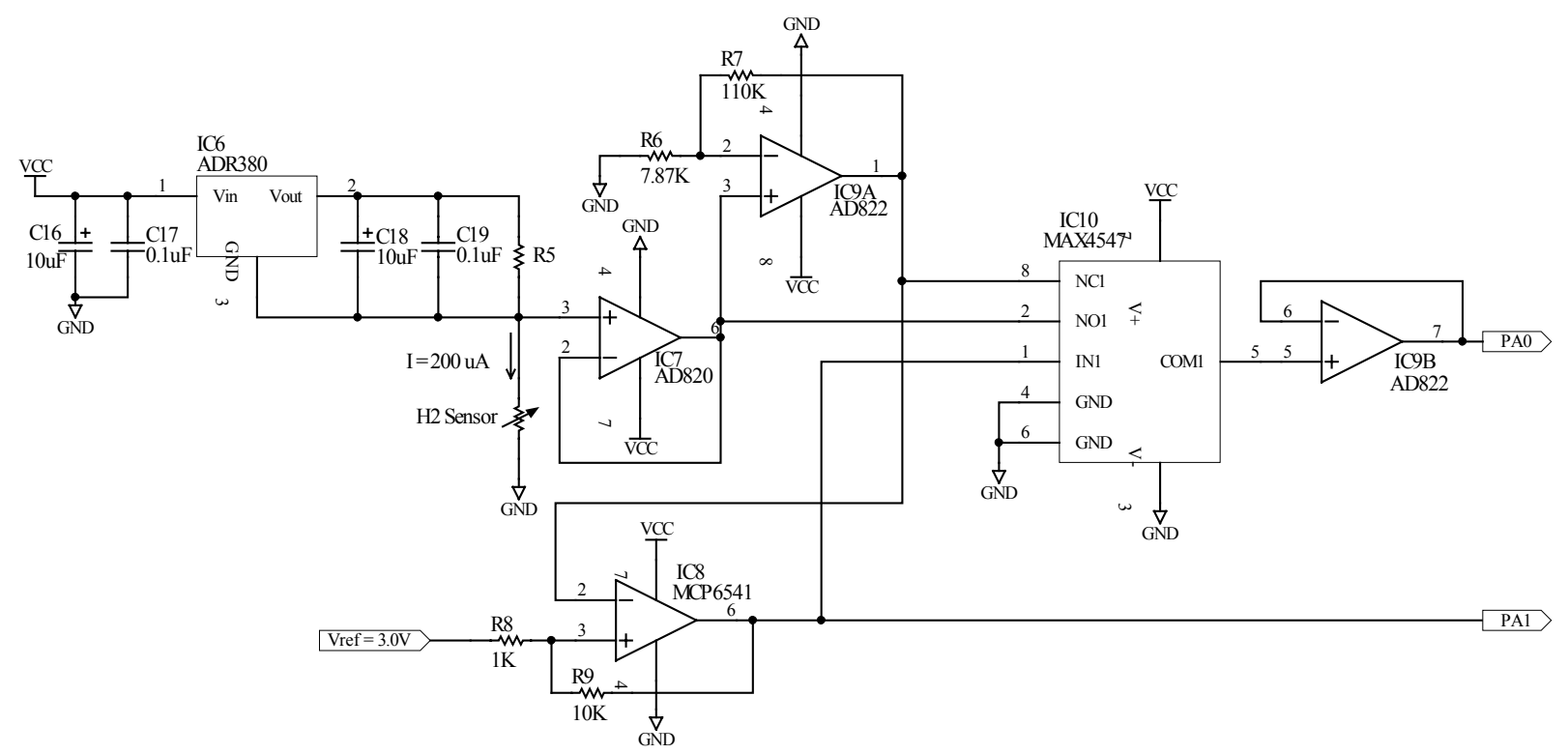

Figure 3. Circuit schematic of the sensor interface. The output (pin 6 of the op amp) is connected to the ADC0 port (pin 40) of the microcontroller AT90S8535 (see Fig. 2).

Figure 3 shows the schematic diagram of the hydrogen sensor interface circuit. The voltage reference IC6 (ADR380) provides a constant voltage of $2 \mathrm{~V}$ across pin 2 and 3 . When R5 is set to $10 \mathrm{k} \Omega$, IC6 provides a constant current of $200 \mu \mathrm{A}$ through the hydrogen sensor [31]. By Ohm's law, the output voltage of the unity-gain op amp IC7 (AD820) $V$ is proportional to the sensor resistance $R_{S}$ :

$$
V=R_{S} \times 200 \mu A
$$

The microcontroller has a 10 bit analog-to-digital converter with a maximum sampling voltage of $3 \mathrm{~V}$. In other words, the sampling voltage from 0 to $3 \mathrm{~V}$ will be linearly converted to a digital value of 0 to 1023 , with a voltage resolution of about $3 \mathrm{mV}$. From Eq. (1), a $3 \mathrm{mV}$ voltage resolution leads to a resistance resolution of $15 \Omega$, which cannot accurately represent the resistance variation of the 
hydrogen sensor when the hydrogen concentration is larger than $100 \mathrm{ppm}$ because the electrical resistance of the sensor becomes less than $60 \Omega$ at $100 \mathrm{ppm}$. To increase the resolution, another op amp, IC9, with a gain of 15 is used to amplify the output voltage from IC7. The outputs from both IC9 (with gain of 15) and IC7 (with gain of 1) are sent to a switch (IC10), which is controlled by a comparator (IC8). When the output of IC7 is between 0 to $200 \mathrm{mV}$ (corresponds to $R_{S}$ of 0 to $1 \mathrm{k} \Omega$ ), the comparator will send an output of 1 to Pin 1 of the switch so the switch can choose IC9 as its input. Conversely, when the voltage output of IC7 is larger than $200 \mathrm{mV}$, the switch will select IC7 as its input. With this design, the sensor interface circuit will generate a voltage of $200 \mathrm{mV}$ to $3 \mathrm{~V}$ when the sensor resistance is from $1 \mathrm{k} \Omega$ to $15 \mathrm{k} \Omega$, and $0 \mathrm{~V}$ to $3 \mathrm{~V}$ when the sensor resistance is 0 to $1 \mathrm{k} \Omega$. As a result, the resistance resolution increases to $1 \Omega$ when the sensor resistance is less than $1 \mathrm{k} \Omega$.

The output of the comparator is also sent to the $\mathrm{I} / \mathrm{O}$ port of the microcontroller (PA1) and is included as a part of the data. When the host computer receives a data package from the sensor node, it will divide the measurements by 15 if the value of PA1 is 1 . Doing so will ensure the measured voltage is linearly proportional to the resistance from 0 to $15 \mathrm{k} \Omega$.

a)
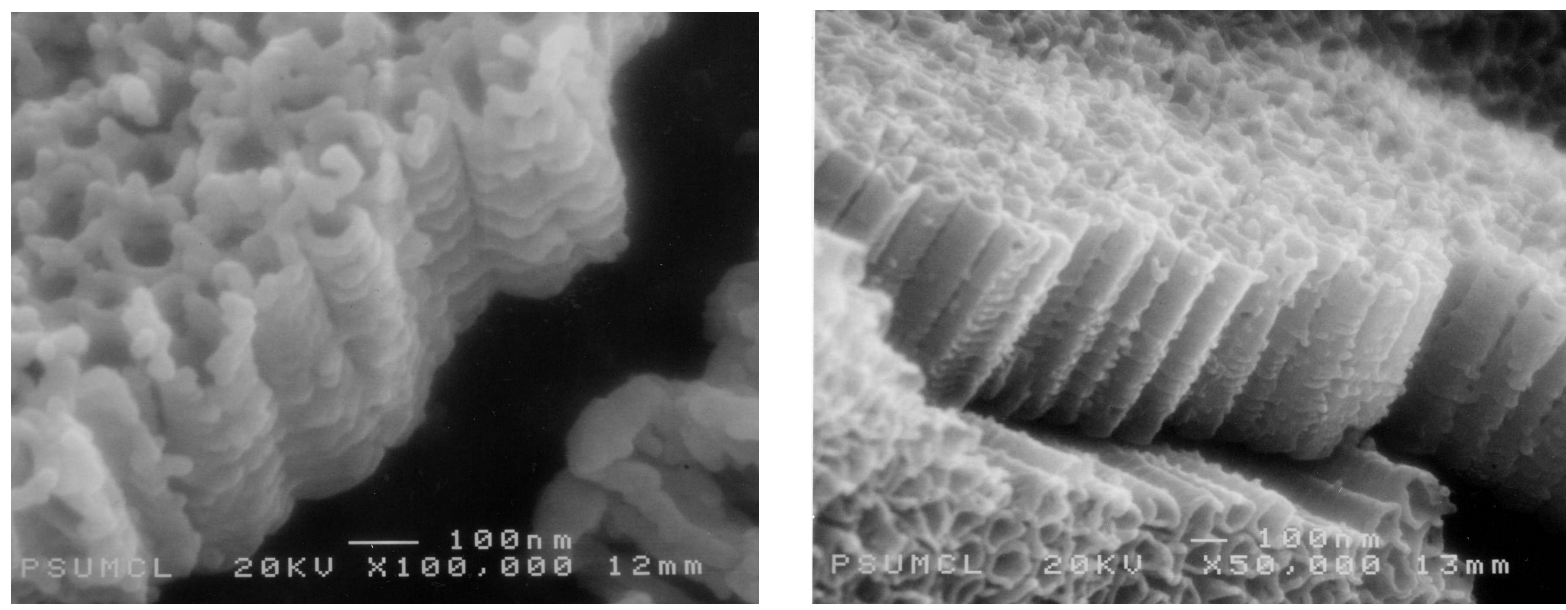

b)
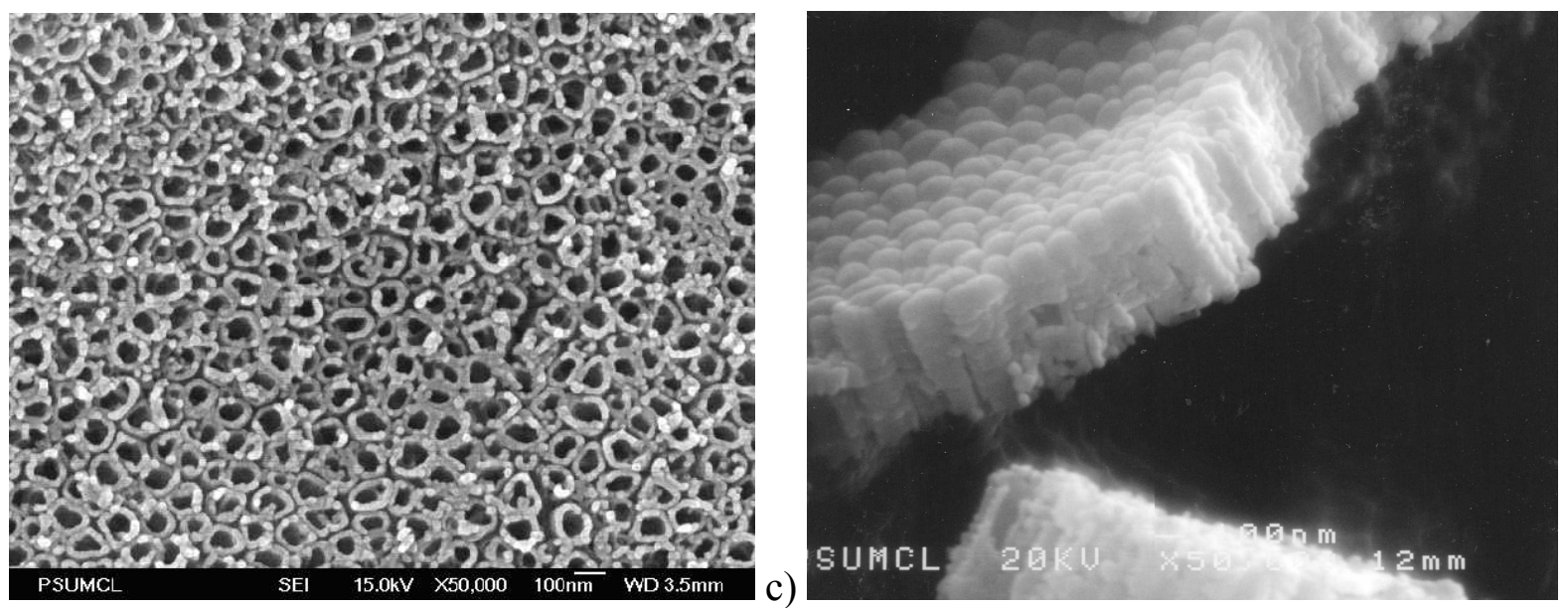

Figure 4. (a) (upper row) Side view of titanium nanotube films; nanotube length is approximately $650 \mathrm{~nm}$. (b) (lower left) SEM image showing the top view of the titania nanotubes. The average tube diameter is $76 \mathrm{~nm}$. (c) (lower right) SEM image showing the insulating barrier layer at the bottom of the nanotubes. 


\section{Fabrication of Titania Nanotube Hydrogen Sensor}

The sheets of highly ordered titania nanotubes used as the hydrogen sensors are made by anodization of titanium foil (99.5\% pure from Alfa Aesar, Ward Hill, MA, USA) of thickness 0.25 $\mathrm{mm}$. The anodization is performed in an electrolyte medium of $0.5 \%$ hydrofluoric acid (J. T. BakerPhillipsburg, NJ, USA) in water, using a platinum foil cathode. In this work $650 \mathrm{~nm}$ long nanotubes were prepared using $20 \mathrm{~V}$-anodization voltage having inner diameters of $76 \mathrm{~nm}$ with standard deviation of $15 \mathrm{~nm}$, wall thickness of $27 \mathrm{~nm}$ with standard deviation of $6 \mathrm{~nm}$. The titanium foil was anodized for 45 minutes, resulting in uniform nanotube arrays grown atop the supporting titanium metal foil, with an electrically insulating barrier layer separating the nanotubes from the conducting titanium foil as shown in Fig. 4. Energy dispersive x-ray analysis and thermogravimetric analysis indicated the as-fabricated nanotubes are amorphous and oxygen-deficient. Annealing studies in oxygen ambient showed no discernible variation in nanotube dimensions to $580^{\circ} \mathrm{C}$, with crystallization in the anatase phase occurring at $280^{\circ} \mathrm{C}$, and transformation to rutile beginning at $430^{\circ} \mathrm{C}$ [29]. Prior to electrical measurements the nanotubes were annealed at $500^{\circ} \mathrm{C}$ for six hours in an oxygen ambient to crystallize the tube walls and improve the stoichiometry. Two platinum contact pads, $10 \mathrm{~mm} \times 2 \mathrm{~mm}$ spaced $2 \mathrm{~mm}$ apart, were deposited on the nanotube array with the electrodes connected to the sensor node to measure the electrical resistance change of the nanotubes.

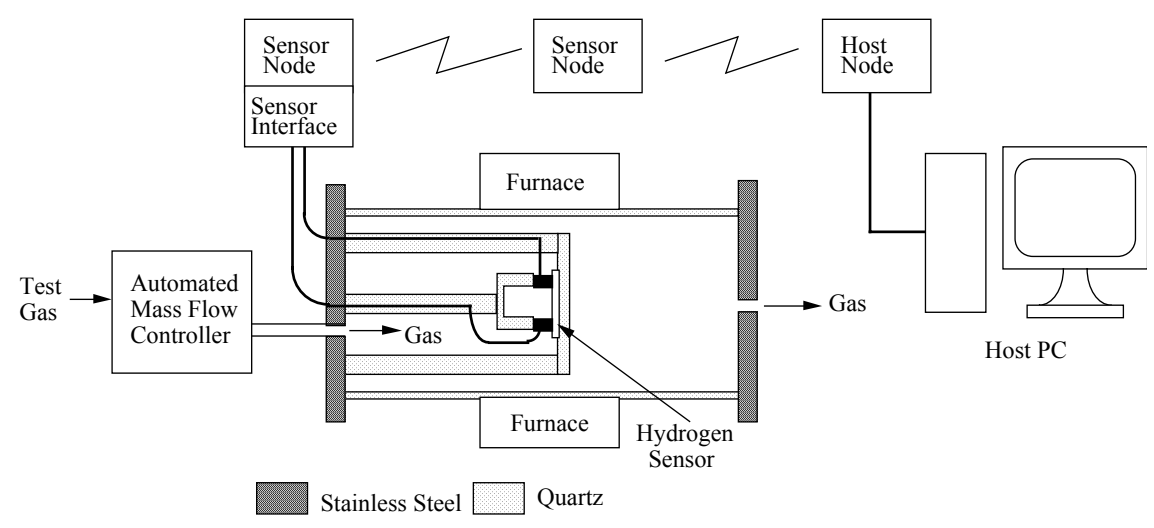

Figure 5. The experimental setup for testing the sensor response at various hydrogen concentrations. For room temperature measurements the sensor and sensor node are placed within the chamber; for elevated temperature measurements only the hydrogen sensor is placed within the test chamber, with an electrical connection passing to the interface circuitry. Using other sensor nodes as data relays the hydrogen measurements are sent to the host node, and there downloaded to the host PC for graphical presentation.

\section{Experiments}

The experimental setup used in these measurements is illustrated in Fig. 5. The titania nanotube hydrogen sensor was placed within a $8 \mathrm{~cm}$ inner diameter $60 \mathrm{~cm}$ long quartz tube furnace that enabled precise control of both ambient temperature and atmosphere. A computer-controlled mass flow controller was used to regulate gas flow through the test chamber, which was kept at 1 atm throughout the experiment. Prior to data collection the test chamber was evacuated using a mechanical pump, 
whereupon nitrogen $(99.999 \%$ pure) was passed while the sensor was heated to the desired temperature. The test gases examined, oxygen, carbon dioxide, ammonia, carbon monoxide and hydrogen, were mixed in appropriate ratios with nitrogen to create the desired test gas ambient.

The titania nanotube sensors show increasing hydrogen sensitivity with increasing temperature [22], therefore in this work all hydrogen measurements were conducted at $290^{\circ} \mathrm{C}$. Figure $6 \mathbf{a}$ shows the change in measured electrical resistance of the $76 \mathrm{~nm}$ diameter nanotube arrays as the hydrogen gas concentration is cycled from $100 \mathrm{ppm}$ to $500 \mathrm{ppm}$ and then back to $100 \mathrm{ppm}$, in $100 \mathrm{ppm}$ steps; note the lack of hysteresis. Figure $\mathbf{6 b}$ shows the measured hydrogen sensitivity of the $76 \mathrm{~nm}$ diameter nanotube array.

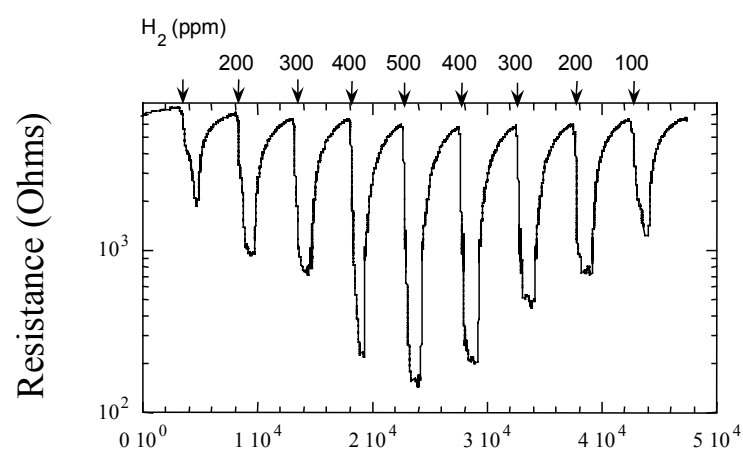

a)
Time (Sec.)

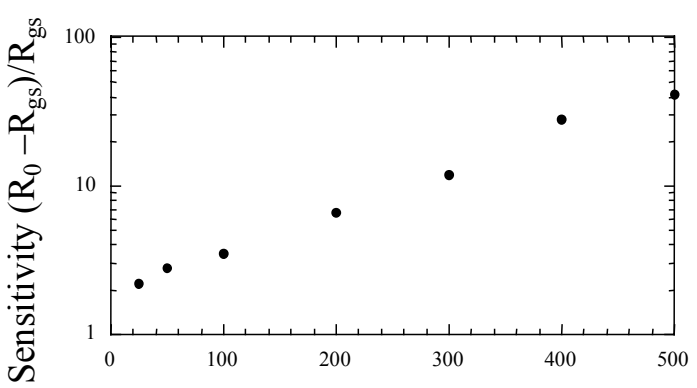

Hydrogen concentration (ppm)

Figure 6. a) Measured electrical resistance of a $76 \mathrm{~nm}$ diameter titania nanotube hydrogen sensor when exposed to different concentrations of hydrogen at $290^{\circ} \mathrm{C}$. The nitrogenhydrogen mixture was passed for $1500 \mathrm{~s}$; the chamber was then flushed with nitrogen for $3000 \mathrm{~s}$ before passing the nitrogen-hydrogen mixture again. b) The hydrogen sensitivity of a $76 \mathrm{~nm}$ titania nanotube sensor at $290^{\circ} \mathrm{C}$ for low hydrogen concentrations. $\mathrm{R}_{\mathrm{o}}$ denotes the electrical resistance of the sensor in a nitrogen environment, $\mathrm{R}_{\mathrm{gs}}$ denotes the steady-state value electrical resistance at the given hydrogen concentration.

Figure 7 shows the nominal physical location of the four sensor nodes used in our experiments. Node 2, connected to the hydrogen sensor kept within the test chamber, transmitted the sensor information to Node 3 since it was the only node within the transmission range of Node 2. Similarly, Node 4 was the parent node of Node 3 due to its proximity, and Node 1 was the parent node of Node 4 .

\section{Results and Discussion}

A $76 \mathrm{~nm}$ diameter titania nanotube hydrogen sensor was placed within the test chamber, held constant at $290^{\circ} \mathrm{C}$, and repeatedly cycled between a pure nitrogen environment and nitrogen with $100 \mathrm{ppm}$ hydrogen, see Figure 8a, and nitrogen to nitrogen with $50 \mathrm{ppm}$ hydrogen as shown in Figure 8b. The pressure in the test chamber was kept constant at one atmosphere. Every two seconds, Node 2 converted the electrical resistance of the hydrogen sensor to voltage, and relayed the information to Node 1 using Node 3 and 4 as relays. The host PC downloaded measurement information from Node 1 every 6 seconds. The host computer also instructed the host node to send out 
a broadcast signal at every 5 minutes to re-established communication links between nodes (which tented to get moved around over the 10 hour measurement period).

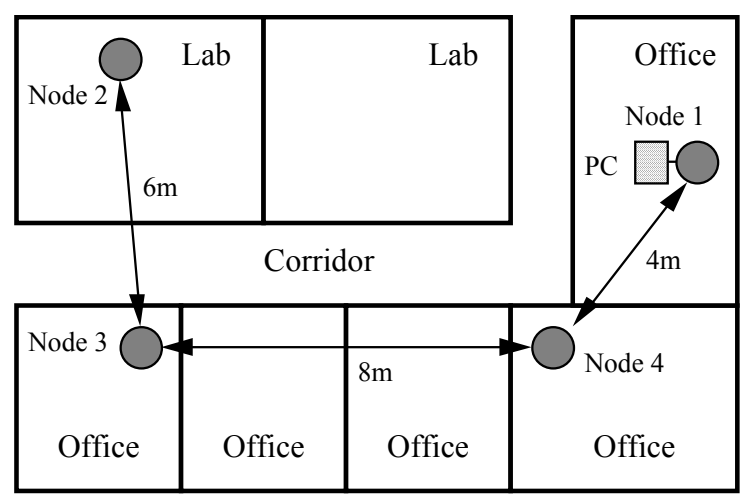

Figure 7. Nominal physical layout of hydrogen sensor network nodes. Node 2 is connected with the hydrogen sensor placed within the test chamber, Node 3 and Node 4 are relays, and Node 1 is the host node; the nodes can of course be re-located within the general area. The described configuration allows Node 3 to relay sensor information for Node 2 and Node 4 for Node 3; for this configuration the communication to Node 2 will be lost if either Node 3 or Node 4 is damaged.

As the sensor is switched from a nitrogen to nitrogen plus 100 ppm hydrogen atmosphere there is a factor of 200 change in measured voltage, and approximately a factor of 60 change in voltage when cycled between nitrogen and nitrogen plus $50 \mathrm{ppm}$, the lower concentration limit of our mass flow controllers. It should be noted that the shape of the response curve in Figure $\mathbf{8 b}$ is determined to a significant but unknown degree by the hydrogen flow rate through the chamber; within practical measurement times we were not able to achieve a steady state response as the flow through the test chamber is non-linear at that low hydrogen concentration.

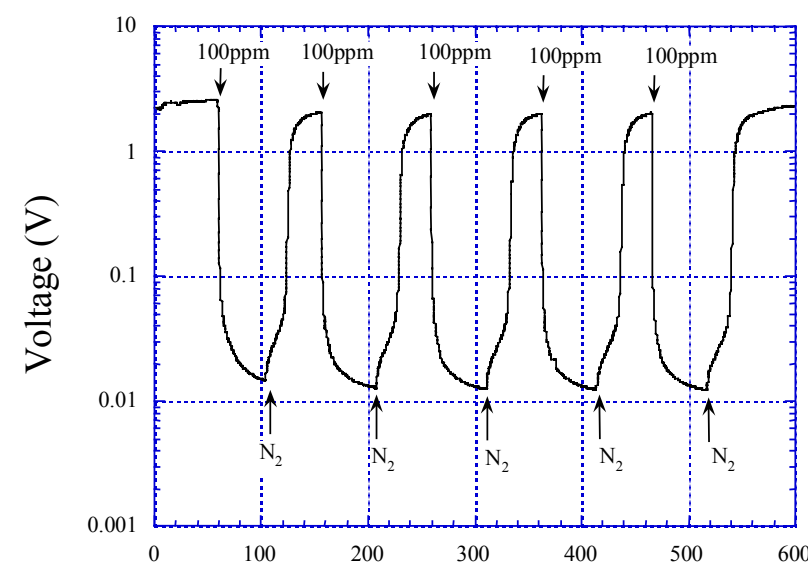

a) Time (Minutes)

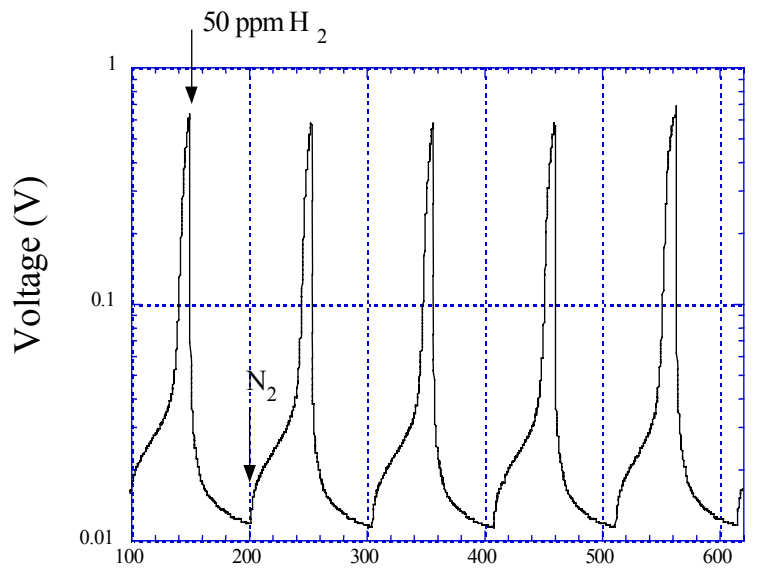

Time (Minutes)

Figure 8. The voltages recorded by the sensor network when the hydrogen concentration is cycled between: (a -left) 0 and $100 \mathrm{ppm}$, and (b-right) 0 and $50 \mathrm{ppm}$. Note for the $50 \mathrm{ppm}$ cycling the nitrogen atmosphere is switched to a nitrogen plus hydrogen atmosphere before a steady state reading is achieved due to inherent difficulties in propagating $50 \mathrm{ppm}$ hydrogen through the test chamber. 
Figure 9a shows the voltage recorded by Node 2 as the concentration of hydrogen is varied from $50 \mathrm{ppm}$ to $10^{4} \mathrm{ppm}$ (steady state values), with Figure 9b showing $50 \mathrm{ppm}$ to $1000 \mathrm{ppm}$. The voltage ranges from $0.049 \mathrm{~V}$ to $2.046 \mathrm{~V}$, linearly corresponding to sensor resistance of $245 \Omega$ to $10.23 \mathrm{k} \Omega$. The behavior of the sensor is consistent, recovering to its original resistance after repeated exposure to varying hydrogen concentrations. The sensor responds linearly to hydrogen concentrations to approximately $500 \mathrm{ppm}$, with an exponential response seen above $500 \mathrm{ppm}$.

Figure 8a shows a factor of 200 change in measured electrical impedance upon exposure to $100 \mathrm{ppm}$ hydrogen. With respect to the ultimate sensitivity we can expect from titania nanotube hydrogen sensors, using resistive bridge circuits changes in electrical resistivity of $1 \%$ can be readily measured. However if we consider a 10\% change in electrical resistance, and linear scaling between hydrogen concentration and electrical resistance as seen in Figure $9 \mathbf{b}$ for low hydrogen concentrations, a hydrogen resolution of 0.05 ppm appears readily achievable.
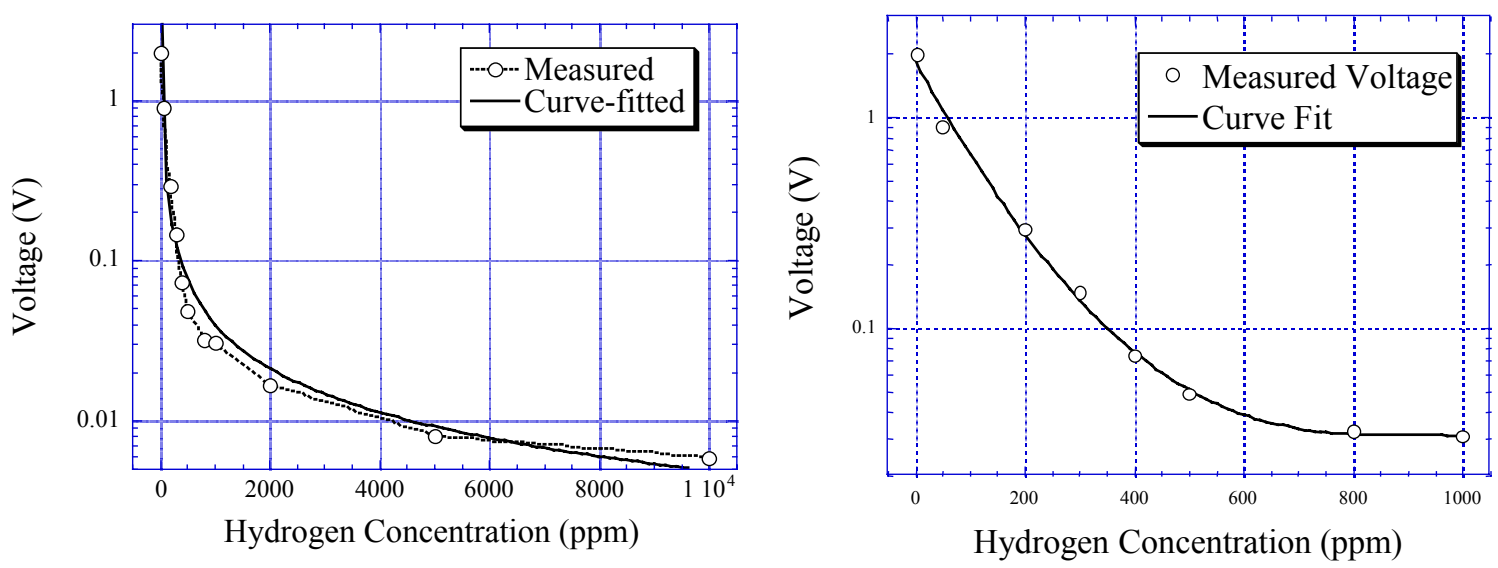

Figure 9. a) The sensitivity of the sensor towards hydrogen. The response is best fitted with a function $V=11.98 \times h^{-0.8375}$, where $V$ is the measured voltage in Volts and $h$ is the hydrogen concentration in ppm. Note: $10,000 \mathrm{ppm}$ is equal to a $1 \%$ hydrogen atmosphere. b) The curve fit on the figure is: $\log V=C_{1} h^{3}+C_{2} h^{2}+C_{3} h+C_{4}$ where $\mathrm{V}$ is the measured voltage in Volts, $h$ is hydrogen concentration in ppm and $C_{1}=-1.22 \times 10^{-9}, C_{2}=4.48 \times 10^{-6}, C_{3}=-5.04 \times 10^{-3}$, and $C_{4}=$ 0.271 .

The change in sensor resistance is likely caused by the hydrogen molecules that are dissociated at the surface of the platinum electrode, which diffuse into the titania lattice where they act as electron donors [32,33] per the 'spill over' model of Roland et al [34]. This chemisorption driven process can change the electrical resistance considerably, and can make the nanotubes sensitive to hydrogen over a wide range of concentrations. Since the sensor completely regains its original resistance with hydrogen cycling it appears that the hydrogen diffusion is confined mostly to the surface layer.

To study the cross sensitivity of the titania nanotube hydrogen sensors they were exposed to oxygen, carbon monoxide, ammonia and carbon dioxide over a range of temperatures. The sensor were found to have no response towards carbon dioxide. The sensitivity of the nanotubes to carbon monoxide and ammonia were negligible compared to that of hydrogen. The resistance of the nanotubes increased in the presence of oxygen, and did not regain their original electrical conductivity even after several hours in a nitrogen environment. 
The sensor network is a robust system, able to function if some of the nodes are damaged or removed. This was demonstrated by both relocating nodes within the network, and removing nodes from the network [35]. For example, Node 3 and Node 4 were arranged so both were within the communication range of Node 2, as shown in Fig. 10. In the beginning of the experiment, Node 2 recognized Node 3 as its parent since it received the broadcast signal from Node 3 before Node 4 . We also noticed Node 2 occasionally switched its parent nodes to Node 4 when the sensor nodes reestablished communications at every 5 minutes. When we turned off Node 3 in the middle of experiment, the host node stopped receiving data from Node 2 since the link was cut off. However, when the host node sent out another broadcast signal, Node 2 reconnected to the network again through Node 4, and the measurement continued. The power of a sensor network is that widespread communication can continue although one or more nodes are removed from the network.

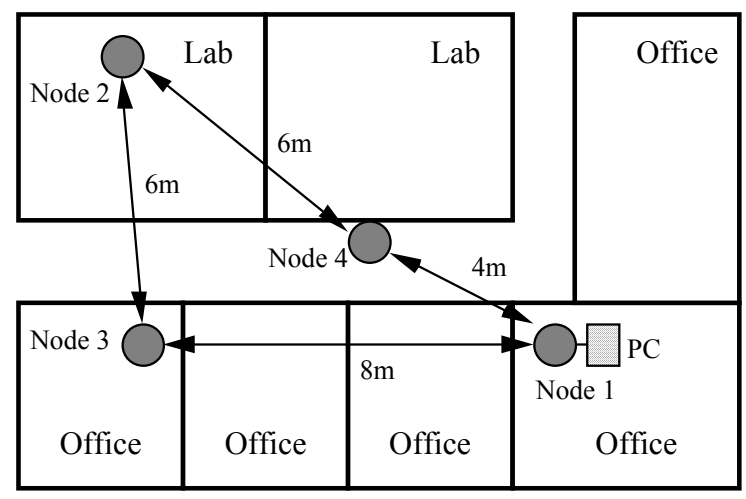

Figure 10. This configuration allows both Nodes 3 and 4 to relay sensor data for Node 2 . Although shorter in communication range, the network can still function even if Node 3 or Node 4 is damaged.

\section{Conclusions}

The application of a wireless sensor network for hydrogen monitoring is presented. The hydrogen sensor network consists of an array of sensor nodes distributed in the area of interest; information from each node is transmitted to a host node using other nodes as relays. A computer is connected to the host node to download and process the measurements of all nodes. The hydrogen sensor is made of titania nanotubes prepared using an anodization voltage of $20 \mathrm{~V}$, resulting in titania nanotubes with inner diameters of $76 \mathrm{~nm}$ and $650 \mathrm{~nm}$ lengths. The sensor shows a resistance variation from $245 \Omega$ to $10.23 \mathrm{k} \Omega$ when cycled between $500 \mathrm{ppm}$ and $0 \mathrm{ppm}$ hydrogen environments. When connected to the sensor node, the sensor-interface circuitry of the node translates the resistance variation of the hydrogen sensor to a voltage change of $0.049 \mathrm{~V}$ to $2.046 \mathrm{~V}$. We have demonstrated the sensor network can still function even when one or more of the relay nodes are damaged, provided there is at least one working node to assume the roles of the damaged nodes.

In our current design, the sensor node can operate for over 100 hours on three AA batteries. In the future, the sensor node can become even more energy efficient by self-engaging low-power mode when idle, and turning on when it only needs to collect and transmit data. This can extend the lifetime of the sensor node to a few months or even years. 
Beyond hydrogen monitoring, the sentinel sensor network can also be applied to other applications by integrating the nodes with different types of sensors. For example, with proper integration of the nodes with desired sensors, the network can be used to monitor for chemical or biological events in airports, theaters, and other public venues. The sensor network can also be used for monitoring of pollution levels, be they in industrial, medical, or household settings.

\section{Acknowledgements}

Xiping Yang is supported by the National Science Foundation under grant ECS-0225346.

\section{References}

1. Rizkalla, S.W.; Luo, J.; Kabir, M.; Chevalier, A.; Pacher, N.; Slama, G. Chronic consumption of fresh but not heated yogurt improves breath-hydrogen status and short-chain fatty acid profiles: a controlled study in healthy men with or without lactose maldigestion. American Journal Of Clinical Nutrition 2000, 72 (6), 1474-1479.

2. Chong, S.K.F.; Ramadan, A.B.; Livesey, E.; Wood, G. The use of a portable breath hydrogen analyser in screening for lactose intolerance in paediatric patients with chronic abdominal pain or chronic diarrhoea. Gastroenterology 2002, 122 (4), M1827 Suppl. 1 APR.

3. Kanabar, D.; Randhawa, M.; Clayton, P. Improvement of symptoms in infant colic following reduction of lactose load with lactase. Journal of Human Nutrition and Dietetics 2001, 14, 359363.

4. Li, D.Y.; Barnes, Y.; Cuffari, C. Who should request a breath hydrogen test? A five year feasibility, sensitivity of clinical suspicion and cost-effectiveness analysis. Gastroenterology 2002, 122, W1144 Suppl. 1.

5. Duro, D.; Rising, R.; Cedillo, M.; et al. Association between infantile colic and carbohydrate malabsorption from fruit juices in infancy. Pediatrics 2002, 109(5), 797-805.

6. Moukarzel, A.A.; Lesicka, H.; Ament, M.E. Irritable bowel syndrome and nonspecific diarrhea in infancy and childhood - Relationship with juice carbohydrate malabsorption. Clinical Pediatrics 2002, 41, 145-150.

7. Lebenthal-Bendor, Y.; Theuer, R.; Lebenthal, A.; Tabi, I.; Lebenthal, E. Malabsorption of modified food starch (acetylated distarch phosphate) in normal infants and in 8-24-month-old toddlers with non-specific diarrhea, as influenced by sorbitol and fructose. Acta Paediatrica 2001, 90(12), 1368-1372.

8. Ledochowski, M.; Widner, B.; Murr, C.; Sperner-Unterweger, B.; Fuchs, D. Fructose malabsorption is associated with decreased plasma tryptophan. Scandinavian Journal Gastroenterology 2001, 4, 367-371.

9. Backus, R.C.; Puryear, L.M.; Crouse, B.A.; Biourge, V.C.; Rogers, Q.R. Breath hydrogen concentrations of cats given commercial canned and extruded diets indicate gastrointestinal microbial activity vary with diet type. Journal of Nutrition 2002, 6, 1763S-1766S Suppl. 2. 
10. Riordan, S.M.; McIver, C.J.; Duncombe, V.M.; Thomas, M.C.; Bolin, T.D. Evaluation of the rice breath hydrogen test for small intestinal bacterial overgrowth. American Journal of Gastroenterology 2000, 95(10), 2858-2864.

11. Bauer, T.M.; Schwacha, H.; Steinbruckner, B.; Brinkmann, F.E.; Ditzen, A.K.; Kist, M.; Blum, H.E. Diagnosis of small intestinal bacterial overgrowth in patients with cirrhosis of the liver: poor performance of the glucose breath hydrogen test. Journal of Hepatology 2000, 33(3), 382-386.

12. Funayama, Y.; Sasaki, I.; Naito, H.; Fukushima, K.; Shibata, C.; Masuko, T.; Takahashi, K.; Ogawa, H.; Sato, S.; Ueno, T.; Noguchi, M.; Hiwatashi, N.; Matsuno, S. Monitoring and antibacterial treatment for postoperative bacterial overgrowth in Crohn's disease, Diseases of The Colon \& Rectum 1999, 42 (8), 1072-1077.

13. Pimentel, M.; Chow, E.J.; Lin, H.C. Comparison of peak breath hydrogen production in patients with irritable bowel syndrome, chronic fatigue syndrome and fibromyalgia. Gastroenterology 2000, 118(4), 2141 Part 1 Suppl. 2.

14. Burge, M.R.; Tuttle, M.S.; Violett, J.L.; Stephenson, C.L.; Schade, D.S. Breath hydrogen testing identifies patients with diabetic gastroparesis. Diabetes Care 2000, 23(6), 860-861.

15. Chiloiro, M.; Darconza, G.; Piccioli, E.; De Carne, M.; Clemente, C.; Riezzo, G. Gastric emptying and orocecal transit time in pregnancy. Journal of Gastroenterology 2001, 36(8), 538-543.

16. Stordal, K.; Nygaard, E.A.; Bentsen, B. Organic abnormalities in recurrent abdominal pain in children. Acta Paediatrica 2001. 90(6), 638-642.

17. Bisquera, J.A.; Cooper, T.R.; Berseth, C.L. Impact of necrotizing enterocolitis on length of stay and hospital charges in very low birth weight infants. Pediatrics 2002,109, 423-428.

18. Engel, R.R.; Virnig, N.L. Origin of mural gas in necrotizing enterocolitis. Pediatric Research 1973, 7, 292A.

19. Godoy, G.; Truss, C.; Philips, J.; et al. Breath hydrogen excretion in infants with necrotizing enterocolitis. Pediatric Research 1986, 20, 348A.

20. Garstin, W.I.H.; Boston, V.E. Sequential assay of expired breath hydrogen as a means of predicting necrotizing enterocolitis in susceptible infants. Pediatric Research 1987, 22, 208-210.

21. Cheu, H.W.; Brown, D.R.; et al. Breath hydrogen excretion as a screening test for the early diagnosis of necrotizing enterocolitis. American J. Diseases of Children 1989. 143, 156-159.

22. Varghese, O.K.; Gong, D.; Paulose, M.; Ong, K.G.; Dickey, E. C.; Grimes, C.A.; Extreme changes in the electrical resistance of titania nanotubes with hydrogen exposure, in press, Advanced Materials, 2003.

23. Varghese, O.K.; Gong, D.; Paulose, M.; Ong, K.G.; Grimes, C.A.; Hydrogen sensing using titania nanotubes, in press, Sensors and Actuators B 2003.

24. Delin, K.A. and Jackson, S.P. Sensor web for in situ exploration of gaseous biosignatures. Proceedings of 2000 IEEE Aerospace Conference, Big Sky, MT March 2000.

25. Qi, H.; Iyengar, S.S.; Chakrabarty, K. Distributed sensor networks - a review of recent research. $J$. Franklin Institute 2001, 338, 655-668.

26. Akyildiz, F.; Su, W.; Sankarasubramaniam, Y.; Cayirci, E. Wireless sensor networks: a survey. Computer Networks 2002, 38, 393-422. 
27. Yang, X.; Ong, K.G.; Dreschel, W.R.; Zeng, K.; Mungle, C.S.; Grimes, C.A. Design of a wireless sensor network for long term, in situ monitoring in an aqueous environment. Sensors 2002, 2, 455 472.

28. Gong, D.; Grimes, C.A.; Varghese, O.K.; Hu, W.; Singh, R. S.; Chen, Z.; Dickey, E. C.; Titanium Oxide Nanotube Arrays Prepared By Anodic Oxidation, Journal of Materials Research 2001, 16, 3331-3334.

29. Varghese, O.K.; Gong, D.; Paulose, M.; Grimes, C.A.; Dickey, E.C. Structural stability of titanium oxide nanotube arrays, Journal of Materials Research, vol. 18, pp. 156-165, 2003.

30. Varghese, O. K.; Grimes, C.A. Metal Oxide Nanoarchitectures for Environmental Sensing, Journal of Nanoscience and Nanotechnology, in press, 2003.

31. http://www.analog.com/UploadedFiles/Datasheets/27775266ADR380 1 $0 . p d f$

32. Bates, J.B.; Wang, J.C.; Perkins, R.A. Mechanisms for hydrogen diffusion in $\mathrm{TiO}_{2}$, Physical Review B 1979, 19, 4130.

33. Hill, G.J. The effect of hydrogen on the electrical properties of rutile. British Journal of Applied Physics 1968, 1, 1151.

34. Roland, U.; Braunschweig T.; F. Roessner, F. On the nature of split-over hydrogen. J. Molecular Catalysis A: Chemical 1997, 127, 61.

35. Estrin, D.; Govindan, R.; Heidemann, J.; Kumar, S. Next century challenges scalable coordination in sensor networks. Proceedings of the ACM IEEE International Conference on Mobile Computing and Networking 1999, 263-270.

Sample Availability: Available from the authors.

(C) 2003 by MDPI (http://www.mdpi.net). Reproduction is permitted for noncommercial purposes. 